\title{
28 Research Square \\ Development of a Patient Safety Care Activity Scale for Clinical Nurses in Korea
}

\section{Ya Ki Yang ( $\nabla$ ykyang@wku.ac.kr)}

Wonkwang University

Research

Keywords: Patient Safety, Safety Management, Activity, Nursing, tool

Posted Date: January 19th, 2021

DOI: https://doi.org/10.21203/rs.3.rs-146004/v1

License: (a) (i) This work is licensed under a Creative Commons Attribution 4.0 International License.

Read Full License 


\section{Abstract}

Introduction: This study aimed to develop a scale measuring the patient safety care activity scale for clinical nurses and to verify validity and reliability it.

Methods: A conceptual framework and construct factors were extracted through extensive literature review and in-depth interviews with nursing professionals. In total, 55 items were derived based on the pilot survey. Data were collected from 428 nurses working at general hospitals and analyzed for verifying the reliability and validity of the scale.

Results: 8 factors containing 44 items were extracted from the exploratory factor analysis to verify the construct validity. The 8 factors were security, patient identification, operation (invasive procedure), medication, blood transfusion, management of infection, management of falls \& sores, management of firefighting. These items were verified by convergent, discriminant, and concurrent validity testing. The internal consistency reliability was acceptable (Cronbach's $\mathrm{a}=.96$ ).

Conclusion: The developed patient safety care activity scale is expected to be used as the tool for the development of theory and improvement of patient safety care activities, the empirical testing for cause and effect of patient safety care activities, the development of interventions, education and training programs for improving patient safety care activities, and indicators for evaluation or accreditation of hospital service quality.

\section{Introduction}

Hospital safety incidents refer to all types of errors, mistakes, and accidents that occur in a hospital regardless of the harm caused on the client [1]. Aging of population, increased prevalence of chronic diseases, advances in medical technology, and establishment of a national health insurance system improved people's access to healthcare services, and as a result, unexpected safety incidents are bound to occur. Failure to promote patient safety in a healthcare facility threatens patients' lives as well as undermining patients' trust in healthcare professionals and facility and prolonging hospital stay, resulting in poor healthcare quality and financial loss [2]. Therefore, ensuring patient safety must be the most important responsibility of healthcare facilities, and nurses should demonstrate clear strategies and objectives to enhance patient safety while providing care [3].

The Institute of Medicine (IOM) report that proposed patient safety incidents as a major culprit deteriorating the quality of healthcare service [4] sparked much interest in healthcare service errors in the field of medicine. South Korea conducts healthcare facility accreditation evaluations, which includes items about patient safety, every three years since 2004, and with an implementation of stricter evaluation criteria pertaining to patient safety from 2007, the evaluation now includes more specific items such as marking of surgical site and patient identification before medication administration and sampling for diagnostic purposes [5]. Recently, there have been more effort to institutionalize patient 
safety, such as increased acquiring of or effort to acquire an accreditation from the Joint Commission International $(\mathrm{JCl})$ among tertiary hospitals.

Safety care activities are the identification, improvement, and prevention of problems or potential problems that may arise during the process of treatment and care [6]. The Joint Commission (TJC) standard is the gold standard in patient safety management in hospital practice, and it consists of items such as accuracy of treatment, efficiency of communication among healthcare professionals, safety of drug use (accurate and safe administration), and reduction of risk of injury from falls. Nursing work is closely related to patient safety in types of work such as infection control, medication administration, fall management, and facility management, and because nurses are the final healthcare providers who can detect and address errors pertinent to medication and treatment [7], nurses' interests and perception of safety care activities are critical factors in improving patient safety.

Nurses, who directly deliver healthcare service to patients, are responsible for a broad scope of work, and safety care activities encompass a variety of areas. Thus, standardized guidelines for and an instrument to assess safety care activities would contribute to enhancing the quality of safety care activities and patient safety outcomes through accurate and consistent management and assessment. However, the currently available instruments to measure safety care activities only contain some of the areas in the healthcare accreditation standard, such as only focusing on infection, communication, falls, medical equipment management, surgery/invasive procedures, staff safety, or fire safety [8], or only partially cover a variety of areas, including falls, participant training, infection, facility inspection, fire, patient identification, communication, medication administration, and blood transfusion [9]. For this reason, it is difficult to directly compare safety care activities, and most available tools are nonstandardized tools with unestablished reliability and validity. Furthermore, the validated tools for medication administration safety [10] and falls and bedsore care [11-13] only assess a single safety care activity. As a result, these tools are significant for patient safety in the corresponding areas but cannot present the level of comprehensive patient safety activities. In addition, studies also measure the awareness of patient safety culture to indirectly propose the level of safety care activities with an argument that individuals with a high awareness of patient safety culture will engage in a high level of safety care activities $[8,9,14]$.

Thus, this study aims to develop and validate an instrument that accurately measures the level of safety care activities of clinical nurses to provide foundational data for continuous improvement of safety care activities.

This study aims to develop and validate an instrument that comprehensively measures safety care activities in order to promote a patient safety culture and safety care activities. The specific objectives are as follows:

- Develop an instrument to measure safety care activities of clinical nurses.

- Test the validity and reliability of the developed Safety Care Activity Scale. 


\section{Methods}

\section{Research design}

This is a methodological study for developing and validating the Safety Care Activity Scale for Clinical Nurses.

\section{Scale development process}

The Safety Care Activity Scale was developed based on the scale development guidelines by DeVellis [15].

\section{1) Step 1: Item generation}

To generate the items for the scale, key concepts and components of safety care activities were examined based on the healthcare organization accreditation criteria and literature review. The subcategories and items were compared among existing literature related to safety care activities, and a focus group interview was conducted on seven nurses with at least two years of experience as nursing staff specializing in patient safety in a hospital. With reference to the criterion to use at least two times more items than the anticipated number in the preliminary tool [16], a total of 80 items were chosen. A total of 12 subscales were chosen: security, patient identification, communication, surgery/invasive procedure, fall, infection, pressure injury, blood transfusion, medication administration, fire safety, facility and medical equipment management, and patient safety reporting system. The appropriateness of the preliminary items were reviewed by two nursing professors and were revised and complemented accordingly.

\section{2) Step 2: Development of the preliminary tool}

\section{(1) Convent validity judgment for the preliminary items}

The validity of the preliminary items for the Safety Care Activity Scale was rated on a 4-point scale, with "highly relevant" (4), "quite relevant" (3), "somewhat relevant" (2), and "not relevant" (1). An expert panel of 10 experts, namely three nursing professors and seven nurses with at least 5 years of experience as nursing staff specializing in patient safety, was selected for the validity judgment [17]. The content validity of the 80 preliminary items of the Safety Care Activity Scale was assessed using item content validity index (I-CVI) and scale content validity index (S-CVI).

The I-CVI asks about the relevance, redundancy, and clarity of each item, and all items with an I-CVI for relevance of below .80 were deleted. The remaining items were assessed for redundancy (I-CVI > .80), and those rated to be redundant were deleted. Items rated to be unclear were revised, and a total of 55 items in 12 subscales were identified. The S-CVI, which refers to the percentage of items rated as 3 or 4 by 10 experts, was .83.

\section{(2) Pilot study}


A pilot study was conducted to examine the comprehensibility and reliability of the validated preliminary items as well as the time required to complete the survey. The pilot study was conducted on 30 staff nurses at W hospital from December 5, 2019 to December 8, 2019. None of items were not answered due to difficulty of understanding. The reliability (Cronbach's a) of the preliminary scale was .99, and none of the items increased the Cronbach's a by .10 or higher when deleted. Further, the correlation between the item and all remaining items ranged from .34-.92, based on which all 55 items were selected to be included in the finalized tool.

\section{3) Step 3: Validation of the preliminary tool}

\section{(1) Participants}

Based on Gorsuch's suggestion that the sample size for factor analysis should be at least 5-10 times larger than the number of items [18], 450 clinical nurses were selected in consideration of potential withdrawals. Nurses working in the insurance review division, health examination center, and outpatient setting without direct contact with patients were excluded.

\section{(2) Ethical considerations and data collection}

The authors informed the participants of the purpose, procedure, and confidentiality of the study to the participants prior to data collection, and an informed consent was obtained from the volunteers. The consent form specified that all personal information obtained will only be used for research purposes and that participants have the freedom to withdraw from the study at any time. This study was approved by the institutional review board (IRB) at W University (IRB No: WKIRB-202002-SB-004) before data collection.

A total of 441 questionnaires were retrieved (98\% retrieval rate), and after excluding questionnaires with careless responses, 428 were included in the analysis. It took about 10-15 minutes to complete the survey, and data were collected from clinical nurses of five general hospitals in J province and G, S, P cities. The questionnaires were distributed and collected by the head of the nursing units in each hospital from January 6,2020 to January 31,2020 . The heads of each nursing unit were informed about the purpose of the study, and data were collected from only those hospitals that provided an informed consent to participate in the study. The questionnaire contained the researchers' contact information and email address so that the participants can direct all of their inquiries about study participation or questionnaire content to the researchers, and to ensure voluntary study participation, the written consent form contained an item about voluntary participation so as to allow nurses to autonomously determine their participation.

\section{(3) Validity testing}

The validity of the preliminary items was tested based on construct validity with exploratory factor analysis (EFA), convergent validity, discriminant validity, and criterion validity. The participants' general characteristics were examined using descriptive statistics, and construct validity was tested using EFA 
with principal component analysis and Varimax rotation. The criterion validity was analyzed using Pearson's correlation coefficients with reference to the Korean modified version of the Hospital Survey on Patient Safety Culture, originally developed by the US Agency for Healthcare Research and Quality [19] and translated and modified into Korean by Kim et al. [20], which is the most widely used instrument to measure safety care activities in Korea.

\section{(4) Reliability testing}

The reliability of the preliminary tool was tested based on Cronbach's a for internal consistency and Spearman-Brown coefficient for split-half reliability.

\section{Results}

\section{Participant characteristics}

The mean age of the participants was 30.42 years, and $98.1 \%$ were female. Highest education was bachelor's degree $(73.4 \%)$, associate degree $(22.9 \%)$, and masters or higher $(3.7 \%)$. The mean total clinical career was 7.91 years, and $30.8 \%$ worked in internal medicine ward. There were more healthcare organizations that have been accredited (93.0\%) than those that have not been accredited $(7.0 \%)$. The majority (93.5\%) of the participants had prior training related to safety care activities, and $99.5 \%$ stated that their hospital has a patient safety division (Table 1).

\section{Validity testing}

\section{1) Construct validity}

Construct validity was tested with item analysis and EFA.

(1) Item analysis

The mean score of each item was 2.47-3.89, with a standard deviation of 0.62-1.06. The absolute value of skewness was 0.01-0.59, and that of kurtosis was 0.01-1.06, which was below 2 and thus was deemed acceptable. The correlation coefficient for each item with the total score was .45-.71, meeting the cutoff of .30 or higher. To examine the floor and ceiling effects, the frequency of the item with the lowest score and that of the item with the highest score was compared against the cutoff of $<30.0 \%$ (Table 2).

\section{2) Exploratory factor analysis (EFA)}

EFA was performed to test the construct validity of the tool. Prior to the EFA, Kaiser-Meyer-Olkin (KMO) and Bartlett test of sphericity were performed to determine the suitability of the data for factor analysis. The KMO value was .93, and Bartlett test of sphericity also confirmed that the data has common factors and is suitable for EFA ( $p<.001)$. 
EFA was performed with varimax rotation to test the construct validity of 55 items. Eight factors had an eigenvalue of 1.0 or higher, with a cumulative total variance explained of $76.0 \%$. While 12 factors theoretically established, only eight were identified. Pressure injury management and fall management emerged as a single factor, and factor analysis was performed again after removing 6 items also loaded under another factor and 5 items with a factor loading of .40 or below. After removing 11 items and conducting factor analysis on 44 items, the KMO value was .93 , and Bartlett test of sphericity led to a $\chi^{2}$ statistic of $21141.70(p<.001)$. Eight factors had an eigenvalue for 44 items of 1.0 or higher, and cumulative variance explained was $76.91 \%$ (Table 3 ).

Factor analysis generated 44 items under 8 factors, and among these factors, those that contain the identical items as that in the preliminary tool development was given the same name as that used in the development stage. Management of falls and management of sores were identified to be a single factor, and thus was named management of falls and sores. Factor analysis confirmed 8 items for safety care activities, 4 items for patient identification, 3 items for operation (invasive procedure), 5 items for medication, 6 items for blood transfusion, 6 items for management of infection, 9 items for management of falls and sores, and 4 items for management of firefighting.

\section{2) Convergent validity and discriminant validity}

To test the convergent validity and discriminant validity of each item, multi trait-multi item matrix analysis was performed. The correlation between each item with their overarching factor met the cutoff of .40 or higher with a range of .77-.94, and so the convergent validity of each item was established. Discriminant validity is deemed established when the difference in the correlation coefficient for the item with its overarching factor and the correlation coefficient for the item with another factor is more than twofold higher than the standard error of the correlation coefficient, and the results confirmed that none of the items markedly deviated from the criterion. Thus, discriminant validity was established (Table 4).

\section{3) Criterion validity}

With reference to the study findings that safety care activities increase with increasing awareness of patient safety culture [8-10,12], the criterion validity was tested with reference to a patient safety culture scale. The Hospital Survey on Patient Safety Culture developed by AHRQ [19] and translated and modified into Korean by Kim et al. (2007) [20] was used, and the scale consists of 44 items in 6 subscales, including 18 items for hospital work environment, 4 items about supervisor/manager's attitude, 6 items about communication, 3 items for frequency of events reported, 1 item for level of general patient safety, 11 items for hospital climate, and 1 item for reported number of incidents. With the exception of the 1 item for reported number of incidents, all items are rated on a 5-point Likert scale. To prevent response bias, negatively worded items were included, which were reverse coded for analysis. A higher score indicates greater awareness of patient safety culture. The reliability (Cronbach's $a$ ) of the scale was .78 and the reliability of each subscale ranged from .67-.84 in the study by Kim et al. (2007) [20]. In this study, the reliability of the entire scale was .84, and that of each subscale ranged from .62-.87. 
There was a statistically significant correlation between awareness of patient safety culture and safety care activities $(r=.51, p<.001)$, and the correlations between patient safety culture and all subscales of safety care activities were also significant (Table 5 ).

\section{Reliability testing}

Internal consistency, as measured with Cronbach's a, was .96, and the Cronbach's a for each subscale was as follows: security .89 , patient identification .91 , operation(invasive procedure) .88 , medication .90 , blood transfusion .94, management of infection .95, management of falls and sores .95, and management of firefighting .94. The cutoff for internal consistency measured with Cronbach's a is .70 or higher for a new instrument and .80 or higher for an existing instrument. None of the items increased the Cronbach's a by .10 or higher when deleted. The coefficient was .80 for the entire items and .70 or higher for all of the subscales, thereby verifying reliability.

\section{Finalized Safety Care Activity Scale}

After empirically establishing the validity of the scale via EFA, the Safety Care Activity Scale was finalized to 44 items in 8 subscales. The rating scale was a 4-point Likert scale from 1 "strongly disagree," 2 "disagree," 3 "agree," and 4 "strongly agree," where a higher score indicates greater compliance with safety care activities. The finalized Safety Care Activity Scale consisted of 7 items for security, 4 items for patient identification, 3 items for operation (invasive procedure), 5 items for medication, 6 items for blood transfusion, 6 items for management of infection, 9 items for management of falls and sores, and 4 items for management of firefighting.

\section{Discussion}

This study was designed to develop an instrument to measure safety care activities of clinical nurses and attempted to present foundational data for improving nursing practice related to patient safety. A standardized patient safety-related nursing activities were identified via a literature review, and the preliminary items for safety care activities were written based on the experiences of healthcare organization accreditation evaluation and current experiences with safety care activities through a focus group interview with 7 clinical nurses who are in charge of patient safety in a general hospital.

The content validity of the preliminary items was tested, and the results showed an I-CVI of .88 and S-CVI of .83 , indicating high validity. The validity was tested in three stages of appropriateness, redundancy, and clarity. The comprehensibility and reliability of the preliminary items and time required to complete the survey were examined through a pilot survey, based on which the preliminary Safety Care Activity Scale was developed. To validate the preliminary tool, the reliability and validity of the tool were tested using a sample consisting of randomly selected clinical nurses from three university hospitals across regions. In terms of the criterion validity, patient safety culture was strongly correlated with safety care activities, and this not only establishes the criterion validity of the developed Safety Care Activity Scale 
but also supports important previous findings that safety care activity increases with increasing awareness of patient safety culture $[8,9,14]$, which adds to the significance of this study.

The reliability of the Safety Care Activity Scale developed in this study as measured with Cronbach's a was .96. Although this cannot be directly compared with previous finding due to the differences in the subscales and items, it is still higher than the Cronbach's a found in other studies that used a safety care activity tool $(.92-.95)$ and is above the criterion of .90 for high reliability for a socio-psychological instrument. Hence, the scale developed in this study can be deemed to have a high reliability.

The finalized Safety Care Activity Scale consisted of 44 items in 8 subscales (security, patient identification, operation(invasive procedure), medication, blood transfusion, management of infection, management of falls and sores, and management of firefighting. A 4-point Likert scale was chosen because using a 3-point or 5-point rating scale may lead to problems related to a neutral category [17]. The finalized tool consisted of a quite large number of items (44), as the study was conducted on nurses in general hospitals that contain a number of units (e.g., medical, surgical, OR, ER, ICU) and not on nurses of a specific unit.

This study proposed a novel factor known as security that is not included in the existing safety care activities. Even if healthcare organizations developed tools and information security guidelines to protect patients' medical information, the security management system may become obsolete if the staff do not actually engage in activities to protect patients' medical information. Hence, security is an essential area to be included in safety care activities in that it allows the prediction of nurses' activities to protect patients' medical information in obligatory environments such as hospitals and help establish policies or guidelines to promote the protection of medical information.

Patient identification is at the basis of all nursing activities, and it included contents about the requirement to identify a patient with two or more pieces of information and the need to implement a rule and regulation to require two healthcare professionals to identify a patient before blood transfusion. This not only satisfies one of the six goals proposed by $\mathrm{JCl}$ (2015), which states that comprehensive effort should be made to develop measures and protocol to identify patients correctly using two identifiers [21] and method and timing of patient identification suggested by the Korea Institute for Healthcare Accreditation (KOIHA) (2014)[22] but also presents the details related to patient identification before blood transfusion, sampling for a test, and giving treatment, rendering the tool as more comprehensive.

Operation (invasive procedure) management had a relatively low impact on the overall safety care activities, but this may be attributable to the fact that many of the nurses were stationed in nonsurgical units. The subscale includes items about patient involvement in marking of surgical site and checklist for verification before and immediately before surgery (invasive procedure).

In Korea, medication errors account for the vast majority of errors in therapeutic nursing activities directly delivered to patients [10]. Approximately $56.2 \%$ of medication errors are made by a nurse [23], and $79 \%$ of these cases were found to be caused by "carelessness," where the error could have been prevented with 
more precaution [24]. The tool developed in this study not only includes the $\mathrm{JCl}$ guidelines about labeling for safe medication administration and developing a list for each patient [21] and the "label high-risk and high-alert medications for storage" in the category "accurate communication among healthcare professionals" proposed by the KOIHA [22] but also classifies the items by specific area, which would help actively prevent errors.

Blood transfusion is closely linked to nurses' roles of patient identification, blood type verification, infusion of correct blood, and monitoring of adverse reactions, and blood transfusion errors can be prevented with nurses' safety management activities [25]. In Korea, the Korean Society of Blood Transfusion developed the guidelines for blood management and blood transfusion in 2002, and healthcare organization accreditation evaluation includes meticulous entry of patient's blood sample information, appropriateness of testing before transfusion, time from dispensing of blood to transfusion, and monitoring of adverse reactions during transfusion in the evaluation of appropriateness of blood transfusion management [22]. The present tool contains contents about monitoring of adverse reactions during blood transfusion and additionally includes measures to be taken upon onset of adverse reactions.

In this study, nurses were found to perceive infection control as the most important. This may be attributable to the increased perception among healthcare providers that infection control is the only way to ensure safety for themselves and patients, owing to the continual exposure to novel infections such as MERS and COVID-19 in recent years. Infection control is also one of the 13 domains and a significant part of the KOIHA [22] evaluation criteria, so this study is significant in that it developed a tool that can be applied to clinical nurses of all areas.

Management of falls and sores has emerged as a serious patient safety-related issue in healthcare organizations $[13,24]$. Falls and pressure injuries are predictable and preventable health problems, as opposed to an unavoidable accident, whose responsibility often falls on nurses [13,26], and so nurses must pay close attention during the duration of the patient's hospital stay. Preventing, as opposed to treating after the fact, falls and pressure injuries is helpful for both patients and caregivers and can contribute to improving the quality of care. The present tool emphasized the importance of prevention by including a more detailed description of fall and pressure injury prevention.

Healthcare facilities are at a high risk for fire due to the numerous small wards with long aisles and builtin combustible materials, such as beddings, medicines, and medical equipment. Moreover, the persons occupying the facilities consist of unspecified users and patients with reduced mobility, which may lead to more serious damages and casualties in a natural or man-caused fire compared to other types of buildings. Nurses must be aware of the shelters within the facility and patients' capability of evacuation in advance such that they can guide people to safely evacuate from the building in case of a fire.

In addition to the subscales included in this tool, a review of existing revealed several other areas related to safety care activities, including transport care, suicide, crisis management, and emergency responses. In this study, areas that are only applicable to specific units or subset of patients were excluded, so these 
areas should be added as needed in subsequent studies. The tool developed in this study should be further modified and complemented to be applicable to nurses of various areas who are in charge of patient safety as the awareness of patient safety increases and improves. Further, subsequent studies may also examine the association with patient safety outcomes, such as the rate of safety incidents.

\section{Conclusions}

In this study, a preliminary tool was developed based on a literature review and expert opinions about safety care activities, and the preliminary tool was validated on nurses of university hospitals to finalize the Safety Care Activity Scale. Use of this tool by clinical nurses in hospitals would help effectively identify the problems related to safety care activities and provide safer care. This study was only conducted on nurses of university hospitals, so subsequent studies should examine all types of healthcare organizations.

\section{Declarations}

\section{Ethics approval and consent to participate}

Approval to conduct the study was granted by the University's Human Research Ethics Committee, Wonkwang University.

\section{Consent for publication}

Not applicable.

\section{Availability of data and materials}

The datasets during and / or analysed during the current study available from the corresponding author on reasonable request.

\section{Competing interests}

Not applicable

\section{Funding}

This work was supported by the National Research Foundation of Korea (NRF) grant funded by the Korea government (MSIT) (2019R1F1A1056424)

\section{Author' contributions}

Study design, data collection, and statistical analyses were performed by YY. First and final drafts of the paper were written by YY. 
Acknowledgements

I would like to thank all participants who participated in this study.

\section{Author details}

Department of Nursing, College of Medicine, Wonkwang University, South Korea.

\section{References}

1. Hwang Y, Kim MY, Kang JS. Perception and performance about patient safety management for hospital nurses. AJMAHS. 2016;6(12):39-54. http://dx.doi.org/10.14257/AJMAHS.2016.12.29

2. Kim MR. Concept analysis of patient safety. J Korean Acad Nurs. 2011;41(1):1-8. https://dx.doi.org/10.4040/jkan.2011.41.1.1

3. Park MJ, Kim IS, Ham YL. Development of a perception of importance on patient safety management scale (PI-PSM) for hospital employee. The Journal of the Korea Contents Association. 2013;13(5):333-341. https://dx.doi.org/10.5392/JKCA.2013.13.05.332

4. Kohn L, Corrigan J, Donaldson M. To err is human: building a safer health system, Institute of Medicine, Washington DC: National Academy Press; 2000.

5. Kang YM. Develop standard regulations and procedures for each medical institution. Seoul: Korea Institute for healthcare Accreditation; 2011 July. Report No.: Koiha-2011-005-1.

6. Choi JH, Lee KM, Lee MA. Relationship between hospital nurses' perceived patient safety culture and their safety care activities. J Korean Acad Fundam Nurs. 2010;17(1):64-72.

7. Glancy GD, Chaimowitz G. The clinical use of risk assessment. Canadian Journal of Psychiatry. 2005;50(1):12-17. https://dx.doi.org/10.1177/070674370505000104

8. Lee NJ, Kim JH. Perception of patient safety culture and safety care activity among nurses in smallmedium sized general hospitals. JKANA. 2011;17(4):462-473.

9. Park SJ, Kang JY, Lee YO. A study on hospital nurses' perception of patient safety culture and safety care activity. The Journal of Korean Critical Care Nursing. 2012;5(1):44-55.

10. Kim YM, Kim SY, Kim MY, Kim JH, Lee SK, Jang MK. Patient safety program and safety culture. JKANA. 2010;16(4):455-465. https://doi.org/10.11111/jkana.2010.16.4.455

11. Kim SH, Kim MH, Jung MH. The level of elderly fall prevention activities \& perception of patient safety culture among university hospital nurses. KSLES. 2013;20(1):81-87.

12. Park EO, Jang IS. Development of the home fall prevention checklist for community-dwelling older adults. The Journal of the Korea Contents Association. 2013;13(5):354-365. https://doi.org/10.5392/JKCA.2013.13.05.354

13. Kim YH, Jang JN, Song MJ, Oh MK, Lee YM. Effect of integrative pressure ulcers nursing program for the prevention and management of pressure ulcers. JKDAS. 2015;17(2):1015-1029. 
14. Lee KH, Lee YS, Park HK, Rhu JO, Byun IS. The influences of the awareness of patient safety culture on safety care activities among operating room nurses. J Korean Clin Nurs Res. 2011;17(2):204-214.

15. Kim HY, Kim HS. Effects of perceived patient safety culture on safety nursing activities in the general hospital nurse's. JKANA. 2011;17(4):413-422.

16. Lee HJ, Song RY, Lee EH, Ahn SH. Research Methods and Critical Appraisal, Seoul: KoonJa; 2017.

17. Lynn MR. Determination and quantification of content validity. Nurs Res. 1986;35(6):382-385.

18. Gorsuch R. Factor analysis. 2nd Ed. Hillsdale, NJ: Psychology Press; 1983.

19. Agency for Healthcare Research and Quality. Hospital Survey on Patient Safety Culture. Rockville: Agency for Healthcare Research and Quality; 2004 [ cited 2020 December 27]. Available from: https://www.ahrq.gov/sites/default/files/wysiwyg/professionals/quality-patientsafety/patientsafetyculture/hospital/userguide/hospcult.pdf

20. Kim JE, Kang MA, An KE, Sung YH. A Survey of Nurses' Perception of Patient Safety Related to Hospital Culture and Reports of Medical Errors. J Korean Clin Nurs Res. 2007;13(3):169-179.

21. The Joint Commission. National Patient Safety Goals. Washington DC: The Joint Commission; 2016 [cited 2020 December 27]. Available from: https://www.jointcommission.org/standards/nationalpatient-safety-goals/

22. Korea Institute for Healthcare Accreditation. Accreditation Program for Healthcare Organizations; 2014 [cited 2020 December 27]. Available from: https://www.koiha.or.kr/web/kr/library/establish_board.do

23. Cho W. A Study on the types and causes of medication errors and related drugs-by analyzing AJNs medication error 73 cases. J Korean Pubilc Health Nurs. 2001;16(1):176-189.

24. Evans D, Hodgkinson B, Lambert L, Wood J. Falls risk factors in the hospital setting: A systematic review. Int J of Nurs Pract. 2001;7:38-45. http:/doi.org/10.1046/j.1440-172x.2001.00269.x

25. Kim KS, Kim JA, Kwon SH, Song MS. Development of the nursing process based performance measurement tool for medication management and blood transfusion $\mathrm{J}$ Korean Clin Nurs Res. 2010;16(1):177-196.

26. Kim CG, Suh MJ. An analysis of fall incidence rate and its related factors of fall in inpatients. Journal of Korean Society of Quality Assurance in Health Care. 2012;9(2): 210-228.

\section{Tables}




\begin{tabular}{|c|c|c|}
\hline Characteristics & Categories & $N(\%)$ \\
\hline \multirow[t]{2}{*}{ Sex } & Male & $8(1.9)$ \\
\hline & Female & $420(98.1)$ \\
\hline \multirow[t]{5}{*}{ Age (year) } & $\leq 25$ & $142(33.2)$ \\
\hline & $26 \sim 30$ & $142(33.2)$ \\
\hline & $31 \sim 35$ & $52(12.1)$ \\
\hline & $36 \sim 40$ & $28(6.5)$ \\
\hline & $\geq 41$ & $64(15.0)$ \\
\hline \multirow[t]{3}{*}{ Education level } & 3-year college & $98(22.9)$ \\
\hline & Bachelor & $314(73.4)$ \\
\hline & $\geq$ Master & $16(3.7)$ \\
\hline \multirow[t]{4}{*}{ Total career (year) } & $\nabla 4$ & $196(45.8)$ \\
\hline & $4 \sim 7$ & $74(17.3)$ \\
\hline & $8 \sim 14$ & $72(16.8)$ \\
\hline & $\geq 15$ & $86(20.1)$ \\
\hline \multirow[t]{4}{*}{ Career present unit (year) } & $凶 2$ & $160(37.4)$ \\
\hline & $2 \sim 3$ & $134(31.3)$ \\
\hline & $4 \sim 6$ & $82(19.2)$ \\
\hline & $\geq 7$ & $52(12.1)$ \\
\hline \multirow[t]{2}{*}{ Patient safety education } & Yes & $400(93.5)$ \\
\hline & No & $28(6.5)$ \\
\hline \multirow[t]{2}{*}{ Experiences on accreditation } & Yes & $398(93.0)$ \\
\hline & No & $30(7.0)$ \\
\hline \multirow[t]{5}{*}{ Type of unit } & Internal medicine ward & $132(30.8)$ \\
\hline & Surgical ward & $96(22.4)$ \\
\hline & Emergency room & $54(12.7)$ \\
\hline & Intensive care unit & 78 (18.2) \\
\hline & Operation room/Delivery room & $68(15.9)$ \\
\hline
\end{tabular}


QI department

Yes

426 (99.5)

No

$2(0.5)$

Page 15/23 


\begin{tabular}{|c|c|c|c|c|c|}
\hline Item No & M & SD & Skewness & Kurtosis & Item-total correlation \\
\hline 1 & 3.36 & 0.56 & -0.15 & -0.76 & .417 \\
\hline 2 & 3.33 & 0.59 & -0.25 & -0.64 & .564 \\
\hline 3 & 3.27 & 0.70 & -0.67 & 0.15 & .565 \\
\hline 4 & 3.40 & 0.60 & -0.57 & 0.12 & .491 \\
\hline 5 & 3.51 & 0.50 & -0.06 & -1.01 & .561 \\
\hline 6 & 3.43 & 0.51 & 0.06 & -1.51 & .614 \\
\hline 7 & 3.38 & 0.51 & 0.26 & -1.41 & .679 \\
\hline 8 & 3.68 & 0.47 & -0.76 & -1.43 & .640 \\
\hline 9 & 3.72 & 0.45 & -0.98 & -1.04 & .582 \\
\hline 10 & 3.67 & 0.47 & -0.74 & -1.46 & .634 \\
\hline 11 & 3.67 & 0.49 & -0.98 & -0.46 & .627 \\
\hline 12 & 3.47 & 0.52 & -0.07 & -1.52 & .674 \\
\hline 13 & 3.23 & 0.76 & -0.87 & 0.55 & .624 \\
\hline 14 & 3.41 & 0.54 & -0.08 & -1.07 & .706 \\
\hline 15 & 3.57 & 0.49 & -0.30 & -1.92 & .738 \\
\hline 16 & 3.56 & 0.58 & -1.21 & 1.94 & .663 \\
\hline 17 & 3.58 & 0.53 & -0.70 & -0.74 & .736 \\
\hline 18 & 3.49 & 0.58 & -0.71 & 0.42 & .640 \\
\hline 19 & 3.48 & 0.55 & -0.59 & 0.30 & .739 \\
\hline 20 & 3.54 & 0.54 & -0.54 & -0.95 & .713 \\
\hline 21 & 3.37 & 0.68 & -0.89 & 0.72 & .663 \\
\hline 22 & 3.46 & 0.53 & -0.14 & -1.34 & .730 \\
\hline 23 & 3.63 & 0.49 & -0.64 & -1.30 & .789 \\
\hline 24 & 3.48 & 0.52 & -0.11 & -1.52 & .777 \\
\hline 25 & 3.57 & 0.55 & -0.77 & -0.49 & .638 \\
\hline 26 & 3.67 & 0.48 & -0.84 & -0.96 & .729 \\
\hline 27 & 3.67 & 0.48 & -0.87 & -0.92 & .715 \\
\hline
\end{tabular}




\begin{tabular}{|c|c|c|c|c|c|}
\hline 28 & 3.66 & 0.48 & -0.82 & -1.01 & .742 \\
\hline 29 & 3.68 & 0.48 & -0.92 & -0.82 & .702 \\
\hline 30 & 3.67 & 0.49 & -0.95 & -0.52 & .653 \\
\hline 31 & 3.66 & 0.49 & -0.93 & -0.57 & .631 \\
\hline 32 & 3.58 & 0.51 & -0.55 & -1.21 & .706 \\
\hline 33 & 3.62 & 0.50 & -0.62 & -1.33 & .716 \\
\hline 34 & 3.56 & 0.52 & -0.45 & -1.32 & .692 \\
\hline 35 & 3.59 & 0.50 & -0.47 & -1.50 & .745 \\
\hline 36 & 3.60 & 0.51 & -0.64 & -1.09 & .790 \\
\hline 37 & 3.58 & 0.54 & -0.79 & -0.51 & .731 \\
\hline 38 & 3.58 & 0.55 & -1.03 & 1.03 & .736 \\
\hline 39 & 3.49 & 0.63 & -1.28 & 2.59 & .731 \\
\hline 40 & 3.55 & 0.55 & -1.05 & 2.01 & .727 \\
\hline 41 & 3.54 & 0.54 & -0.70 & 0.46 & .740 \\
\hline 42 & 3.51 & 0.55 & -0.65 & 0.37 & .731 \\
\hline 43 & 3.57 & 0.54 & -0.73 & -0.61 & .689 \\
\hline 44 & 3.59 & 0.49 & -0.36 & -1.88 & .764 \\
\hline 45 & 3.53 & 0.54 & -0.57 & -0.83 & .693 \\
\hline 46 & 3.53 & 0.51 & -0.24 & -1.68 & .709 \\
\hline 47 & 3.23 & 0.60 & -0.14 & -0.49 & .561 \\
\hline 48 & 3.27 & 0.58 & -0.11 & -0.50 & .570 \\
\hline 49 & 3.14 & 0.66 & -0.16 & -0.72 & .568 \\
\hline 50 & 3.20 & 0.61 & -0.14 & -0.49 & .565 \\
\hline 51 & 3.48 & 0.52 & -0.11 & -1.52 & .742 \\
\hline 52 & 3.54 & 0.50 & -0.17 & -1.98 & .762 \\
\hline 53 & 3.49 & 0.50 & 0.06 & -1.01 & .777 \\
\hline 54 & 3.43 & 0.52 & -0.03 & -1.34 & .671 \\
\hline 55 & 3.46 & 0.52 & -0.04 & -1.52 & .716 \\
\hline
\end{tabular}


Page 18/23 


\begin{tabular}{|c|c|c|c|c|c|c|c|c|c|}
\hline \multirow{2}{*}{ Item No. } & \multirow{2}{*}{ Communality } & \multicolumn{8}{|c|}{ Factors } \\
\hline & & 1 & 2 & 3 & 4 & 5 & 6 & 7 & 8 \\
\hline 1 & .508 & .048 & .255 & .567 & -.122 & -.020 & .092 & .118 & .286 \\
\hline 2 & .744 & .122 & .011 & .777 & .193 & .117 & .012 & .073 & .263 \\
\hline 3 & .629 & .045 & .027 & .666 & .297 & .187 & .221 & .046 & .092 \\
\hline 4 & .607 & .088 & .122 & .707 & .029 & .150 & .240 & -.052 & .029 \\
\hline 5 & .707 & .201 & .038 & .754 & .102 & .149 & .225 & .094 & -.070 \\
\hline 6 & .740 & .213 & .149 & .777 & .091 & .123 & .091 & .195 & .005 \\
\hline 7 & .757 & .226 & .032 & .710 & .097 & .262 & .172 & .253 & .171 \\
\hline 8 & .831 & .197 & .155 & .238 & .120 & .081 & .785 & .199 & .187 \\
\hline 9 & .871 & .202 & .189 & .239 & .122 & .013 & .845 & .0410 & .084 \\
\hline 10 & .882 & .119 & .165 & .317 & .159 & .077 & .806 & .0990 & .224 \\
\hline 11 & .624 & .154 & .409 & .245 & .190 & .073 & .552 & .0450 & .155 \\
\hline 16 & .829 & .364 & .113 & .152 & .094 & .152 & .285 & .120 & .730 \\
\hline 17 & .822 & .306 & .194 & .242 & .118 & .160 & .328 & .210 & .664 \\
\hline 18 & .679 & .272 & .153 & .196 & .256 & .247 & .171 & -.018 & .622 \\
\hline 20 & .814 & .091 & .400 & .289 & .206 & .121 & .077 & .602 & .370 \\
\hline 21 & .759 & .07 & .212 & .244 & .211 & .207 & .100 & .599 & .439 \\
\hline 22 & .762 & .329 & .202 & .096 & .335 & .179 & .223 & .633 & .094 \\
\hline 23 & .793 & .354 & .509 & .215 & .168 & .153 & .189 & .518 & .088 \\
\hline 25 & .738 & .424 & .221 & .163 & .313 & -.014 & .034 & .613 & -.088 \\
\hline 26 & .768 & .432 & .582 & 0.15 & .169 & -.007 & .250 & .356 & .046 \\
\hline 27 & .765 & .276 & .644 & .114 & .311 & -.028 & .248 & .280 & .155 \\
\hline 28 & .833 & .224 & .712 & .136 & .356 & .115 & .298 & .098 & .136 \\
\hline 29 & .790 & .303 & .700 & .122 & .333 & .056 & .266 & .029 & .089 \\
\hline 30 & .821 & .228 & .810 & .081 & .241 & .108 & .057 & .133 & .125 \\
\hline 31 & .789 & .289 & .787 & .057 & .217 & .073 & .050 & .156 & .053 \\
\hline 32 & .787 & .216 & .392 & .134 & .707 & .132 & .091 & .161 & .132 \\
\hline
\end{tabular}




\begin{tabular}{|c|c|c|c|c|c|c|c|c|c|}
\hline 33 & .861 & .243 & .313 & .150 & .794 & .116 & .121 & .123 & .093 \\
\hline 34 & .844 & .265 & .197 & .187 & .797 & .045 & .164 & .184 & .045 \\
\hline 35 & .761 & .295 & .324 & .031 & .619 & .187 & .192 & .267 & .203 \\
\hline 36 & .818 & .370 & .468 & .068 & .570 & .144 & .190 & .175 & .215 \\
\hline 37 & .708 & .362 & .334 & .143 & .601 & .111 & .096 & .225 & .106 \\
\hline 38 & .666 & .588 & .278 & .195 & .266 & .106 & .028 & .207 & .282 \\
\hline 39 & .783 & .722 & .213 & .165 & .061 & .142 & .102 & .165 & .357 \\
\hline 40 & .750 & .743 & .203 & .092 & .207 & .117 & .203 & .099 & .204 \\
\hline 41 & .786 & .768 & .173 & .150 & .257 & .120 & .182 & .089 & .146 \\
\hline 42 & .779 & .745 & .143 & .161 & .281 & .197 & .073 & .022 & .232 \\
\hline 43 & .758 & .687 & .387 & .088 & -.044 & .132 & .164 & .260 & .122 \\
\hline 44 & .747 & .642 & .406 & .152 & .196 & .163 & .167 & .232 & .020 \\
\hline 45 & .693 & .621 & .121 & .280 & .397 & .234 & .003 & .044 & -.013 \\
\hline 46 & .768 & .778 & .213 & .108 & .220 & .175 & .133 & .091 & .018 \\
\hline 47 & .912 & .212 & .113 & .170 & .076 & .901 & .090 & .014 & .022 \\
\hline 48 & .881 & .294 & .083 & .117 & .096 & .864 & .064 & -.028 & .109 \\
\hline 49 & .835 & .128 & .029 & .275 & .162 & .823 & -.028 & .160 & .108 \\
\hline 50 & .865 & .091 & .063 & .197 & .072 & .859 & .074 & .174 & .186 \\
\hline \multicolumn{2}{|c|}{ Eigen value } & 20.71 & 3.70 & 2.72 & 1.94 & 1.74 & 1.47 & 1.31 & 1.03 \\
\hline \multicolumn{2}{|c|}{ Explained variance (\%) } & 15.31 & 12.02 & 10.75 & 10.36 & 8.38 & 7.64 & 6.62 & 5.83 \\
\hline \multicolumn{2}{|c|}{$\begin{array}{l}\text { Cumulative explained } \\
\text { variance (\%) }\end{array}$} & 15.31 & 27.34 & 38.08 & 48.44 & 56.82 & 64.46 & 71.08 & 76.91 \\
\hline \multicolumn{10}{|c|}{$\mathrm{KMO}=.93$, Bartlett's test: $\chi^{2}=21141.70(p<.001)$} \\
\hline
\end{tabular}


Table 4. Multi-Trait/Multi-ltem Matrix for Item Convergent and Item Discriminant $(n=428)$

Factor

Item Correlation between each item and total scores of

$2 * \mathrm{SE}$

No sub-factor

$\begin{array}{llllllll}\text { F1 } & \text { F2 } & \text { F3 } & \text { F4 } & \text { F5 } & \text { F6 } & \text { F7 } & \text { F8 }\end{array}$

Security

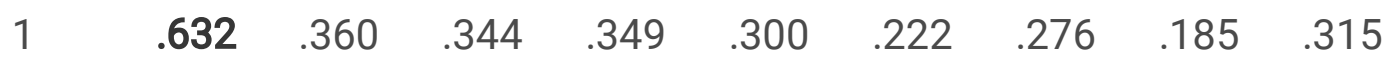

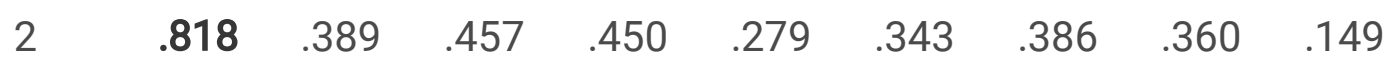

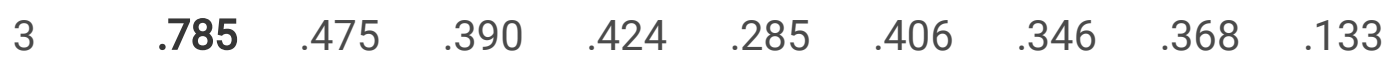

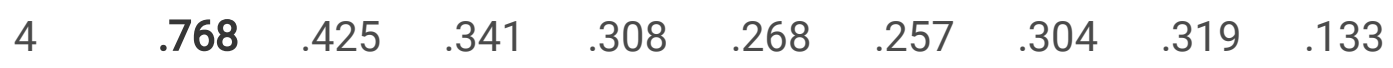

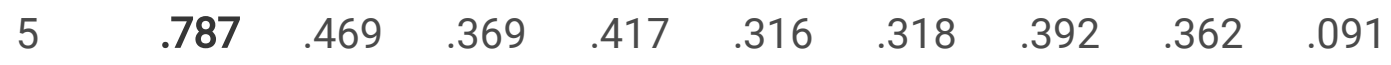

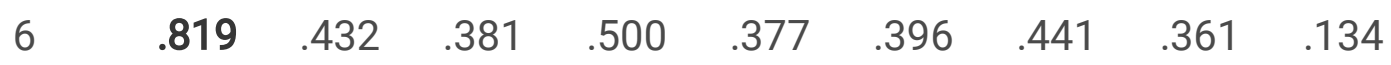

$\begin{array}{llllllllll}7 & .836 & .502 & .496 & .560 & .344 & .421 & .494 & .492 & .143\end{array}$

$\begin{array}{lllllllllll}\text { Patient identification } & 8 & .502 & .898 & .566 & .499 & .505 & .454 & .480 & .266 & .088\end{array}$

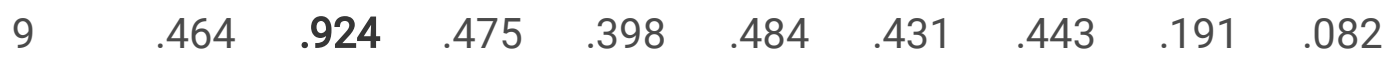

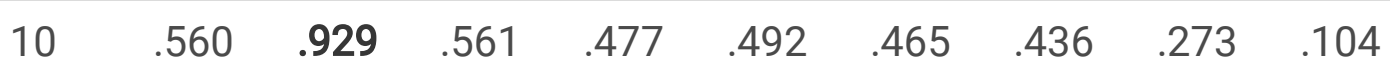

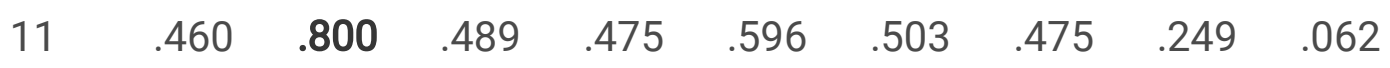

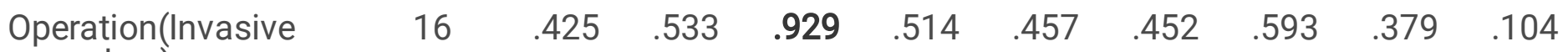

procedure)

\begin{tabular}{|lllllllllll|}
\hline & 17 & .520 & .604 & .928 & .611 & .537 & .518 & .599 & .401 & .114 \\
\hline Medication & 18 & .435 & .462 & .846 & .452 & .443 & .497 & .542 & .429 & .076 \\
\hline & 20 & .503 & .462 & .565 & .855 & .633 & .576 & .509 & .343 & .123 \\
\hline & 21 & .477 & .430 & .553 & .819 & .493 & .532 & .472 & .394 & .123 \\
\hline & 22 & .395 & .468 & .490 & .850 & .595 & .648 & .609 & .373 & .077 \\
\hline & 23 & .527 & .463 & .433 & .811 & .636 & .676 & .697 & .369 & .081 \\
\hline Blood transfusion & 25 & .348 & .328 & .331 & .776 & .562 & .602 & .592 & .219 & .049 \\
\hline & 26 & .398 & .537 & .495 & .687 & .847 & .635 & .680 & .236 & .078 \\
\hline & 27 & .362 & .546 & .505 & .683 & .885 & .696 & .602 & .207 & .071 \\
\hline 2 & .392 & .609 & .510 & .632 & .906 & .720 & .604 & .316 & .079 \\
\hline 2 & .364 & .553 & .468 & .565 & .883 & .690 & .621 & .258 & .082 \\
\hline 30 & .294 & .434 & .422 & .579 & .876 & .640 & .569 & .269 & .059 \\
\hline 31 & .268 & .409 & .387 & .558 & .858 & .626 & .585 & .234 & .060
\end{tabular}


Management of infection

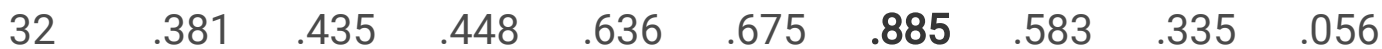

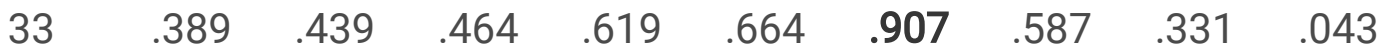

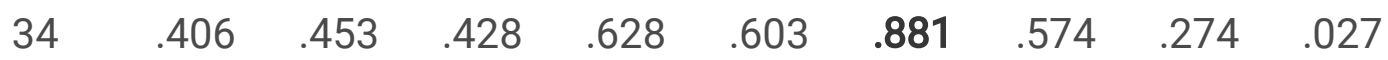

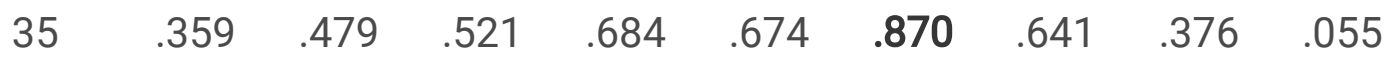

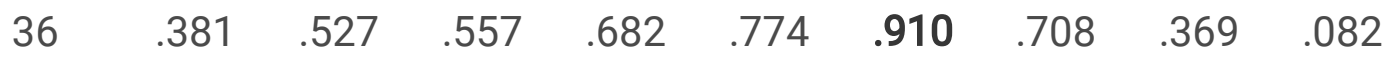

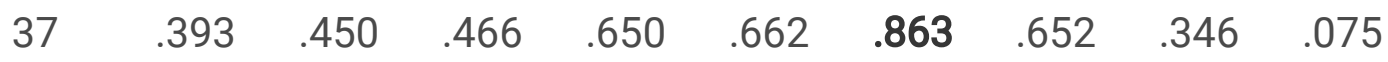

$\begin{array}{lllllllllll}\text { Management of falls \& } & 38 & .422 & .422 & .582 & .617 & .615 & .639 & .793 & .374 & .070\end{array}$ sores

$\begin{array}{llllllllll}39 & .424 & .445 & .628 & .595 & .539 & .528 & .861 & .390 & .096\end{array}$

$\begin{array}{llllllllll}40 & .369 & .476 & .563 & .557 & .577 & .599 & .854 & .360 & .082\end{array}$

$\begin{array}{llllllllll}41 & .406 & .471 & .545 & .590 & .584 & .613 & .873 & .373 & .068\end{array}$

$\begin{array}{llllllllll}42 & .404 & .398 & .579 & .548 & .537 & .603 & .860 & .434 & .060\end{array}$

$\begin{array}{llllllllll}43 & .341 & .455 & .515 & .581 & .629 & .493 & .813 & .343 & .061\end{array}$

$\begin{array}{llllllllll}44 & .424 & .477 & .487 & .647 & .698 & .652 & .830 & .383 & .083\end{array}$

$\begin{array}{llllllllll}45 & .448 & .343 & .427 & .530 & .497 & .601 & .766 & .446 & .061\end{array}$

$\begin{array}{llllllllll}46 & .358 & .400 & .469 & .547 & .572 & .583 & .860 & .379 & .051\end{array}$

Management of fire fighting

$\begin{array}{llllllllll}47 & .397 & .268 & .384 & .340 & .284 & .345 & .436 & .943 & .042\end{array}$

$\begin{array}{llllllllll}48 & .361 & .251 & .441 & .326 & .283 & .365 & .489 & .922 & .035\end{array}$

$\begin{array}{llllllllll}49 & .462 & .227 & .386 & .435 & .250 & .364 & .407 & .910 & .074\end{array}$

$\begin{array}{llllllllll}50 & .427 & .280 & .446 & .426 & .254 & .336 & .386 & .921 & .084\end{array}$

S.E $=$ Standard Error. 
Table 5. Correlation between Patient Safety Culture and Patient Safety Nursing Activity Instrument $(n=428)$

\begin{tabular}{|c|c|c|c|c|c|c|}
\hline \multicolumn{7}{|c|}{ Patient Safety Culture } \\
\hline Total & 口 & 口 & 口 & 口 & प & 0 \\
\hline
\end{tabular}

\begin{tabular}{|c|c|c|c|c|c|c|c|c|}
\hline \multirow[t]{9}{*}{ Patient Safety Nursing Activity } & Total & $.51^{\star *}$ & $.48^{\star \star}$ & $.43^{\star \star}$ & $.44^{\star \star}$ & $.20^{\star *}$ & $-.30^{\star \star}$ & $.22^{\star \star}$ \\
\hline & 口 & $.49^{\star \star}$ & $.43^{\star *}$ & $.41^{\star \star}$ & $.50^{\star \star}$ & $.13^{* *}$ & $-.35^{\star \star}$ & $.38^{\star \star}$ \\
\hline & [ & $.39^{* \star}$ & $.33^{\star *}$ & $.36^{* *}$ & $.40^{\star \star}$ & $.13^{\star *}$ & $-.28^{\star \star}$ & $.28^{\star *}$ \\
\hline & [ & $.43^{\star \star}$ & $.40^{\star *}$ & $.37^{\star \star}$ & $.43^{\star \star}$ & $.14^{\star \star}$ & $-.30^{\star \star}$ & $.30^{\star \star}$ \\
\hline & ( & $.44^{\star \star}$ & $.78^{\star \star}$ & $.46^{\star *}$ & .36 & $.41^{\star \star}$ & $-.31^{\star \star}$ & $.31^{\star \star}$ \\
\hline & ૫ & $.43^{\star \star}$ & $.81^{\star \star}$ & $.43^{\star *}$ & .38 & $.50^{\star \star}$ & $-.37^{\star \star}$ & $.39^{\star \star}$ \\
\hline & [ & $.44^{* *}$ & $.39^{\star \star}$ & $.35^{\star \star}$ & .40 & $.21^{* *}$ & $-.28^{\star \star}$ & $.31^{\star \star}$ \\
\hline & [ & $.45^{\star \star}$ & $.39^{\star \star}$ & $.36^{\star \star}$ & .39 & $.22^{\star \star}$ & $-.28^{\star \star}$ & $.31^{\star \star}$ \\
\hline & ] & $.48^{\star \star}$ & $.46^{\star \star}$ & $.43^{\star \star}$ & .37 & $.33^{\star *}$ & $-.35^{\star \star}$ & $.34^{\star \star}$ \\
\hline
\end{tabular}

** $p<.001$

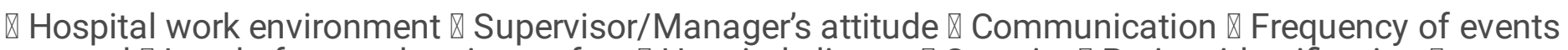
reported $\otimes$ Level of general patient safety $\otimes$ Hospital climate $\otimes$ Security $\otimes$ Patient identification $\otimes$ Operation(Invasive procedure) $\otimes$ Medication $\otimes$ Blood transfusion $\otimes$ Management of infection $\otimes$ Management falls \& sores $\otimes$ Management of fire fighting 